\title{
"Family planning will mean that there will not be any babies" - Knowledge, beliefs, and acceptance of contraception among South Sudanese women
}

\author{
Kelly Ackerson ${ }^{1 *}$ and Ruth Zielinski ${ }^{2}$ \\ ${ }^{1}$ Western Michigan University, Bronson School of Nursing, Michigan, United States \\ ${ }^{2}$ University of Michigan, School of Nursing, MI, United States
}

\begin{abstract}
Objective: Explore knowledge, beliefs and acceptance of modern family planning methods among South Sudanese women.

Methods: Ancillary analysis of qualitative data gathered from field notes taken during six Home Based Life-Saving Skills workshops conducted between 2013 and 2017. Participant responses recorded verbatim and analyzed through content analysis.

Results: Sixty-eight women participated in discussions. An overarching theme "double-edged sword" evolved from the narratives. Upheaval from villages in South Sudan to refugee camps in northern Uganda has changed reproductive health norms with favorable and unfavorable consequences. Within that theme, three sub-themes evolved, cultural norms, changing times, and desire to learn. Cultural norms were to abstain from sex while breastfeeding 2 to 3 years, considered the one acceptable family planning method. Women had very little knowledge of modern contraception, including condoms, but desired to learn and teach others. Participants recognized traditional ways of child spacing and avoiding pregnancy were no longer sufficient and contraceptive methods (outside of abstinence) were important to prevent mistimed pregnancy.
\end{abstract}

Conclusions: Efforts to increase knowledge, understanding and use of modern family planning methods must be sensitive to cultural norms.

\section{Introduction}

Maternal mortality remains appallingly high in sub-Saharan Africa with 546 maternal deaths per 100,000 live births [1]. Reasons for high rates of maternal deaths include poverty, malnutrition, lack of access to quality healthcare facilities, high rates of fertility, and pregnancy at early ages [2]. One approach to improving survival and quality of life for women of childbearing age is utilization of modern methods of family planning. Consistent use of contraception in women of reproductive age decreases maternal and infant deaths by spacing pregnancies, reducing adolescent pregnancies, and decreasing overall fertility [3].

Despite the benefits, rates of contraception use are typically low among populations with scarce resources, such as refugees living in crisis-affected areas [4]. Thirty percent of the world's refugee population resides in Africa, many in the sub-Saharan region. The current conflict in South Sudan resulted in over 2 million people, many of whom are women, fleeing the country [5]. As of December 2017, over 1 million refugees from South Sudan are estimated to be residing in refugee camps in Northern Uganda [5]. While actual rates of contraception use among South Sudanese refugee women living in northern Uganda is unknown, given that rates in other sub-Saharan refugee populations are low, it is likely that rates in this population would be similarly low.

Utilizing qualitative data collected during birth attendant training sessions undertaken first in South Sudan and subsequently in refugee camps located in northern Uganda, the purpose of this study was to explore knowledge, beliefs and acceptance of modern family planning methods among South Sudanese women.

\section{Methods}

This was a planned ancillary analysis of qualitative data gathered from extensive field notes taken during six Home Based Life-Saving Skills (HBLSS) workshops with South Sudanese women as participants conducted between 2013 and 2017 (Table 1). Developed by the American College of Nurse-Midwives (ACNM), HBLSS is a picturebased program of community education that focuses on identifying and preventing problems during pregnancy and birth [6]. Content is presented using an introductory vignette that invites storytelling and discussion followed by demonstration and practice. Pictorial takeaction cards are used throughout the workshops and upon completion, each participant is given a booklet containing these take-action cards with the expectation that the information is shared with the community. Literacy is not required to participate in the workshop. Experienced nurse-midwives with training in HBLSS conducted the workshops.

Correspondence to: Kelly Ackerson, Western Michigan University, Bronson School of Nursing, 1903 West Michigan Avenue, Kalamazoo, Michigan 490085345, United States, E-mail: kelly.ackerson@wmich.edu

Key words: family planning, contraception, South Sudanese, Uganda, refugee

Received: February 28, 2018; Accepted: March 16, 2018; Published: March 20, 2018 
Table 1. Participants by training session date \& location.

\begin{tabular}{|c|c|c|}
\hline Year & Location & $\begin{array}{c}\text { Number of women } \\
\text { participating }\end{array}$ \\
\hline 2013 & Kiir Village, South Sudan & 8 \\
\hline 2015 & Nyumanzi refugee camp, Uganda & 9 \\
\hline 2016 & Olua refugee camp, Uganda & 13 \\
\hline 2016 & Mungula refugee camp, Uganda & 12 \\
\hline 2017 & Olua refugee camp, Uganda & 14 \\
\hline 2017 & Mungula refugee camp, Uganda & 12 \\
\hline TOTAL & & 68 \\
\hline
\end{tabular}

\section{HBLSS family planning content}

One of the HBLSS sessions, titled "Many children or many pregnancies", relates to issues of family planning and child spacing. During this session, problems related to inadequate family planning such as pregnancy at an early age, short pre-pregnancy intervals and too many pregnancies are introduced and discussed. Participants are encouraged to discuss their familiarity with family planning methods as well as utilization within their community. In communities where condoms and other forms of contraception are available, participants are offered an opportunity to learn more through hands-on practice. These discussions offered the opportunity to better understand the knowledge, acceptability and potential barriers to uptake of modern family planning methods within the community.

\section{Participant recruitment and characteristics}

This report includes data from a total of 6 training sessions conducted between 2013 and 2017. Sixty-eight women, all South Sudanese, participated in the training and contributed to the information gathered in the field notes. Women chosen to participate in the training were living in the communities and all expressed a desire to participate. Some participants had functioned as traditional birth attendants and others were church lay leaders, reported ages ranged from 30 to mid-60s, all were either married or widowed and all had children.

\section{Data collection}

The length of the workshops ranged from 3 to 5 days, with the session specific to family planning encompassing approximately 3 hours of the total workshop time. Prior to the first workshop, we sought to find a female interpreter, as our assumption was participants would not be comfortable discussing sensitive topics with a male interpreter. However, we were unsuccessful and a South Sudanese male interpreter who was well known to the community was utilized. We found women freely shared their knowledge and opinions, which included challenging the prevailing gender and cultural norms. We continued to use this interpreter, as he is fluent in Dinka and English. Utilization of the same interpreter for all sessions contributed to consistency with both the information shared in the workshop and data collected in field notes.

Because questions and responses were interpreted word for word, the time needed for interpreting allowed for the participants' responses to be written down verbatim. After each day of the training session, debriefing occurred whereby those conducting the training sessions reviewed the notes and clarified missing or confusing information.

\section{Qualitative data analysis}

This planned ancillary analysis was determined by both authors' respective universities' Institutional Review Boards to be non- regulated. Data were analyzed and reported in aggregate and only involved information that cannot be directly or indirectly linked to a specific individual.

Qualitative data from each set of field notes were merged into one data set. Merged data included information gathered from participant responses related to child spacing, family planning and contraception. To ensure trustworthiness, each researcher conducted a content analysis separately, focusing the analysis on text related to family planning. A qualitative descriptive approach was utilized whereby the data is presented in the women's words with minimal inference of meaning or interpretation by the researchers [7]. Data were organized thematically by the individual researchers who then met and discussed themes and interpretation of meaning until consensus was reached. Field note texts were then re-read to confirm that the themes represented the data.

\section{Results}

In each of the 6 workshops, all of the women participated in the discussions and provided stories. A majority of the participants had arrived in the camps since the most recent conflict in 2013, fleeing from rural villages in South Sudan. Two women had been in the camps since an earlier conflict in the 1990s. Participants freely offered prior knowledge or opinions about family planning use in their culture and indicated a desire to learn more about contraception, including practicing with locally available condoms on bananas.

Overwhelmingly, participants indicated that use of modern family planning methods was not the current acceptable cultural norm within their community. However, some expressed concern that leaving the relative isolation of villages in rural South Sudan for more densely populated refugee camps in northern Uganda would, over time, result in cultural norm changes.

If you don't have a way to get in to the villages easily then these things don't come to the village.

An overarching theme emerged from the data regarding attitudes and beliefs toward family planning we termed a "Double Edged Sword". In other words, women acknowledged that the upheaval from villages to refugee camps has resulted in changes to reproductive health, with favorable and unfavorable consequences. Within this overarching theme, three subthemes evolved, (1) cultural norms, (2) changing times, and (3) desire to learn.

\section{Cultural norms}

Use of condoms or other contraception was regarded negatively as a sign of women's loose behavior. Participants expressed a desire to learn about condoms but also expressed concern:

\section{If I tell women about condoms they will think I am using them.}

If a woman's husband finds condoms in the house, he will assume his wife is sleeping around.

While there was acknowledgement that times may be changing, women indicated that this cultural norm remained for their daughters.

We think it's ok for others to use condoms but as mothers we tell our daughters abstain from sex.

Use of family planning methods to decrease family size was viewed as contrary to cultural norms as offspring are viewed as blessings.

There is concern that family planning will mean that there will not be any babies. 
Ackerson K (2018) "Family planning will mean that there will not be any babies" - Knowledge, beliefs, and acceptance of contraception among South Sudanese women

\section{It is better to die than to use protection; what if the whole population} dies?

With the high infant and child mortality rate, more pregnancies are seen as a requirement to assure some children will survive to adulthood.

We are having more babies because we have a higher rate of death of our children. The causes are war, poverty, many of them will die.

If you have many children then at the end of the day you will have children.

Participants understood that having children closely spaced was unhealthy, indicating that a natural spacing of children of 2-3 years is expected. This is to be achieved through abstaining from sex with your husband while you are breastfeeding and not by using modern family planning methods.

Women are not supposed to "stay" [have sex] with their husbands while they are breastfeeding and they are supposed to breastfeed for 2 years.

Women need to abstain [from sex] during breastfeeding otherwise the baby might get sick and die.

We allow our children to breastfeed for 2-3 years and you don't lie with your husband. That alone is family planning.

After hearing a vignette about a woman who had closely spaced children who became ill and died during pregnancy, participants consistently responded that it was the woman's fault. She should not have allowed her husband to have sex with her, although they acknowledged that it was difficult for a woman to rebuff her husband's advances.

She needs advice, family planning, not to react to her husband at night.

For 2-3 years the husband should "stay away". Three years for a mother and baby, that is the best way for families.

This kind of spacing [less than two years], it is not good, not enough time to raise them, to give them to eat what they are supposed to eat. No time to raise them.

Participants agreed that sex while breastfeeding is taboo, because the breastfeeding infant will become ill. In their culture, abstinence was often achieved by the mother and infant moving to her mother's home. If a woman gets pregnant within the 2-year expectation of abstinence while breastfeeding, there were socially delivered penalties to the woman as described in these stories:

A woman got pregnant while she was breastfeeding; it is the belief that the one that is already born will die. The milk is contaminated. So they took the child away from the mother...they thought she [mother] wanted to kill the child.

One of the women delivered in January, got pregnant in May. All the children in the community also got sick. Other women beat her. She was very traumatized.

Women described polygamy as a commonly practiced cultural norm whereby men can have more than one wife, but women are expected to have only one husband. Men do not have similar social expectations of abstinence during pregnancy or breastfeeding. Participants indicated that it was difficult, if not impossible to control the urges of men.
In our community it is very difficult; men don't want to be told what to do and men are not normally faithful.

Men have more than one wife (up to 6), but women never have more than one husband.

Polygamy was viewed as a mechanism by which children could be spaced because husbands had other wives to go to during the time of breastfeeding. However, participants recognized that there were negative consequences related to men having sex with multiple women.

One way (to avoid infection) is to be faithful-not to allow men to move around with other women. It is possible but it is the normal routine that women remain with one husband but the man may have to have more than one wife.

\section{Changing times}

Participants understood that the cultural norms and traditions were changing due to outside influences. Children may have different attitudes and behaviors and not follow the traditional ways, in part because they were more educated and socialized to different opportunities. Women did not view this as entirely favorable.

Our kids have bigger thinking, a bigger brain, they meet kids from different parts of the world and this has influenced their behavior.

If girls go (away) for education they hear about these things everywhere.

Although abstinence while breastfeeding was an expected behavior, the women recognized that the culture was changing with the younger generation. The women indicated that additional methods of contraception were important to protect the health of the infant and prevent mistimed pregnancies.

Previously we stuck to breastfeeding but new ways are to come. We cannot rule out a mistake - someone will get pregnant while breastfeeding. This must be taken into consideration and precautions taken.

Traditionally, the Dinka women will breastfeed for 2-3 years; they don't have to "stay" with a man (no sex). Now it is different, different styles of lying (sex).

Talking about alternative ways to prevent pregnancy was not easy for the women, since learning about modern family planning methods goes against their beliefs.

We appreciate being trained on planning, but it is very hard to apply. It is hard culturally, but we will try to discuss these options [contraception] with the young women.

Women expressed concern that there were not enough babies being born since they have been in the refugee camps.

People aren't producing as much because they are on the run.

Every Sunday they are only having one child being blessed, which is not normal; it's a big camp.

One reason for this decline is that the current conflict in South Sudan has resulted in many families being separated. There are essentially no employment opportunities within the refugee camps so often men either remain in South Sudan to work or seek employment elsewhere.

A concerning trend discussed by the participants and confirmed in reports [8], is that girls are marrying at a younger age. It is not 
uncommon for girls to be married at age 15 or younger and pregnant shortly after.

Used to be women were more mature when married and had children. Now marrying too young... as young as 15 years. Early marriage causes many weaknesses.

Women are a lot younger when they have children, which contributes to a lot of sickness.

One girl was 14 years old and the girl just neglected her child and went to skip rope with the other children.

When asked why girls were married at such a young age, women stated that traditional beliefs involving stages in a girl's development and rituals that were supposed to be done before she could be married had been abandoned.

There are certain stages you have to go through before you can talk about marriage,

When women first begin to bleed they were not supposed to be around cows or the milk would go bad; they had a lot of rituals that had to be completed before she could be around milk or cows and then get married and that would delay the time of marriage. If they hastened this then the "blood would not have cooled" and the first child would not live.

The dispelling of this cultural belief through modernization, along with financial hardships, has resulted in families selling their daughters into marriage at a younger age.

\section{Desire to Learn}

These women had very little experience with modern family planning methods and very little contraceptive knowledge. Although some were familiar with condoms due to volunteer organizations such as the Red Cross visiting the camps, many of the women had no knowledge. However, all were interested in learning:

I've never seen a condom before today; we cannot say if our children will practice the same; we abstain, our husbands abstain but our children are in a different world. We would love for you to show us; we won't use them but we want to learn.

Women were eager for the chance to learn about family planning, not to change their own reproductive behavior/choices, but so they could to teach others. There was an understanding that times were changing, and they needed to learn:

The use of condoms is so new, especially to our ways of managing affairs. I have been attending training since 1999 and this is important that you are showing all of us this now because you never know who will need this.

Despite not being entirely comfortable with the topic of contraception, the participants asked many questions and desired more knowledge that they could share with others in the community.

At the end of the day, is it better to use a condom, injections, pills?

Concern was expressed regarding the safety of contraception, particularly if it were used improperly, which motivated them to ask questions and learn more about methods of family planning.

Culturally, we do not have condoms, if not used properly and man goes into woman what will happen?

Will have to take a pill every day? What if we run out?
Although the custom was to abstain from sexual intercourse while breastfeeding, they also knew that not all women and men followed this cultural norm.

How do you help them to avoid pregnancy when they are still breastfeeding? If they can't control themselves then we should help them to not get pregnant.

The women's concerns were not only about pregnancy occurring while breastfeeding; they also voiced concerns about a woman's health and the risks inherent in childbirth, particularly for women who were very young, had many pregnancies, or who were in their later reproductive years. They wanted to know how to prevent mistimed pregnancies to benefit the health of these women. We learned it is not uncommon for women to "have 12-13 children, until they have no periods".

How do you stop them [adolescents] from having them [babies]? Closely spaced pregnancy is too much.

Marrying a girl who is too young, older women having lots of children. A Dinka man may be older and he will marry a very young girl, she often dies of complications in delivery.

Some women are too old; an old woman gave birth and was unable to care for her baby. That was the 12th baby. The baby was not well, was not normal. She (woman) was very weak, very thin and it was hard for her to deliver.

Women expressed a desire to move away from cultural norms where the responsibility for controlling fertility rests solely on the women. The women believed that having men take part in learning about family planning would be a benefit.

It can't be just one partner; it is both the husband and wife... he could help to not have so many young babies.

The solution is too slow; we need to get information to everybody. Settings like this help. Some of the men who attend this course will apply the principles - to not marry too young. Make sure to keep the health of the older women by not having too many babies. The community needs to be aware of these problems/fears for older women and younger women. Men need to attend these programs.

\section{Discussion}

The purpose of this study was to explore the knowledge, beliefs and acceptance of modern family planning methods among South Sudanese women. While data specific to use of modern methods of contraception were not collected, conversations in the workshops revealed that use of contraception is not currently culturally acceptable. Very few participants knew about modern methods of contraception and while some had heard of condoms, most had no prior knowledge or education regarding condom use. There were clear cultural taboos regarding use of contraception, that only "loose" women would use condoms and that using contraception would decrease the population. Despite this, women were interested in learning and even sharing the information with others. Women acknowledged that times were changing and that their children would know about family planning methods, motivating them to also learn.

Breastfeeding was the primary method of family spacing, although in their culture this was accomplished not through lactation amenorrhea, but rather because women were not supposed to have sex while breastfeeding. While frequent, on-demand breastfeeding (lactation amenorrhea) suppresses ovulation and protects against 

women

pregnancy, the efficacy of lactation amenorrhea as contraception decreases over time [9]. The timeframe for efficacy varies depending on individual physiology and frequency of lactation [10]. Abandoning the cultural norm of abstaining during breastfeeding will result in shorter child spacing intervals if effective contraceptive methods are not utilized.

Participants understood that the younger generation is becoming more educated, worldly and therefore, more likely to utilize modern methods of contraception. For this reason, women wanted to learn so that they were not left in the dark about what their children were doing. While they acknowledged that times were changing, they did not believe that it was acceptable for their own daughters to have sex outside of marriage or to use contraception.

Currently, the fertility rate for South Sudanese women is one of the highest at over 5 live births per woman [11]. While women acknowledged that having children when too young, too close together or when they were older was not desirable, they maintained that a large number of children were needed so that some would survive. While reducing unplanned pregnancies is a key developmental goal of the Sustainable Development Goals, in this community a concomitant effort is needed to improve the health of women during pregnancy, provide safe childbirth and care for infants after birth so that more infants survive.

An additional factor in preventing pregnancy is empowerment of women within the community. Some women, especially those girls who are married at a young age $(<18)$, are not empowered to say no to marriage and are at a higher risk for pregnancy complications [12]. Married women are expected to submit to their husbands within patriarchal societies with high levels of male control [13]. Women suggested men be a part of the workshop so they too can learn about reproductive health and modern family planning methods.

\section{Limitations}

Data collected were from a subset of the population of South Sudanese refugees, all of whom were married or widowed women and primarily from the Dinka tribe. South Sudan is diverse with over 60 ethnic groups; therefore, the knowledge and attitudes of these women may be different from other ethnic groups. We did not include young, unmarried women in the workshops and their beliefs may be quite different; in addition, men in the community cannot be assumed to have the same attitudes or beliefs regarding modern methods of family planning.

\section{Conclusion}

Maternal death rates are among the highest in South Sudan and while displacement in refugee camps in northern Uganda may offer better healthcare access, there are challenges to consider in efforts to make pregnancy and birth safer for women living in these camps. Increasing utilization of family planning could decrease the rate of adolescent pregnancy, provide spacing between pregnancies and also help women avoid too many pregnancies or childbearing at an older age. Breastfeeding as it is currently practiced (for 2-3 years) provides some measure of family spacing, whether through abstinence as is culturally expected or lactation amenorrhea. Breastfeeding should be encouraged and supported for all the benefits it offers both mother and child. However, as in the stories told, women who get pregnant while breastfeeding are judged harshly, and the child who is breastfeeding is often taken away from the mother which is both tragic and detrimental to the health of the child as well as the mother.
Findings from this study indicate education is needed to address family planning acceptance in order to increase use among South Sudanese refugees. Culturally acceptable educational tools, similar to HBLSS but within a larger framework of reproductive health that includes more information regarding modern contraception methods, are needed. Participants expressed a desire for more knowledge and stressed that the workshops should also be conducted with men. Efforts to increase use of family planning must be sensitive to cultural norms; otherwise they will be unsuccessful and potentially viewed as an effort to control the population of South Sudanese. In addition, societal influences regarding contraception and fertility must be taken into account. Therefore, including community leaders is essential.

Ethical approval: "All procedures performed in studies involving human participants were in accordance with the ethical standards of the institutional and/or national research committee and with the 1964 Helsinki declaration and its later amendments or comparable ethical standards."

\section{References}

1. WHO | Trends in maternal mortality: 1990 to 2015. WHO. 2016. http://www.who.int reproductivehealth/publications/monitoring/maternal-mortality-2015/en/. Accessed February 19, 2018.

2. Firoz T, Chou D, von Dadelszen P, Agrawal P, Vanderkruik R, et al. (2013) Measuring maternal health: Focus on maternal morbidity. Bull World Health Organ 91: 794-796. [Crossref]

3. Mbizvo MT, Chou D, Shaw D (2013) Today's evidence, tomorrow's agenda: Implementation of strategies to improve global reproductive health. Int J Gynecol Obstet 121: S3-S8. [Crossref]

4. Ackerson K, Zielinski R (2017) Factors influencing use of family planning in women living in crisis affected areas of Sub-Saharan Africa: A review of the literature. Midwifery 54: 35-60. [Crossref]

5. UNHCR. UNHCR - Figures at a Glance. unhcr.org. http://www.unhcr.org/en-us/ figures-at-a-glance.html. Published 2017. Accessed February 13, 2018.

6. Sibley L, Buffington ST, Beck D, Armbruster D (2001) Home based life saving skills: promoting safe motherhood through innovative community-based interventions. $J$ Midwifery Womens Health 46: 258-266.

7. Sandelowski M (2000) Whatever happened to qualitative description? Res Nurs Health 23: 334-340. [Crossref]

8. CARE. Inequality and injustice: The deteriorating situation for women and girls in South Sudan's war A Progressive Gender (2013) https://reliefweb.int/sites/reliefweb. int/files/resources/CARE_Inequality-and-injustice_South-Sudan-progressive-genderanalysis_Dec-2016.pdf. Accessed February 13, 2018.

9. Van der Wijden C, Manion C (2015) Lactational amenorrhoea method for family planning. Cochrane Database Syst Rev 10: CD001329. [Crossref]

10. Kennedy KI, Visness CM (1992) Contraceptive efficacy of lactational amenorrhoea Lancet 339: 227-230. [Crossref]

11. Central Intelligence Agency. No Title. https://www.cia.gov/library/publications/ resources/the-world-factbook/geos/od.html. Published 2017.

12. Chantrapanichkul P, Chawanpaiboon S (2013) Adverse pregnancy outcomes in case involving extremely young maternal age. Int J Gynecol Obstet 120: 160-164. [Crossref]

13. Abiola IA, Jolaade AT (2018) Patriarchy and customary law as major cogs in the whee of women's peace building in South Sudan. http://www.ingentaconnect.com/content/ sabinet/aajgida/2016/00000005/00000001/art00004?crawler=true.

Copyright: (C)2018 Ackerson K. This is an open-access article distributed under the terms of the Creative Commons Attribution License, which permits unrestricted use, distribution, and reproduction in any medium, provided the original author and source are credited. 